\title{
Controlling Self-Organizing Dynamics on Networks Using Models that Self-Organize
}

\author{
Pierre-André Noël, ${ }^{*}$ Charles D. Brummitt, and Raissa M. D’Souza ${ }^{\dagger}$ \\ University of California, Davis, California 95616, USA \\ (Received 3 May 2013; published 12 August 2013)
}

\begin{abstract}
Controlling self-organizing systems is challenging because the system responds to the controller. Here, we develop a model that captures the essential self-organizing mechanisms of Bak-Tang-Wiesenfeld (BTW) sandpiles on networks, a self-organized critical (SOC) system. This model enables studying a simple control scheme that determines the frequency of cascades and that shapes systemic risk. We show that optimal strategies exist for generic cost functions and that controlling a subcritical system may drive it to criticality. This approach could enable controlling other self-organizing systems.
\end{abstract}

DOI: 10.1103/PhysRevLett.111.078701

Complex, self-organizing systems are challenging to control because their feedback mechanisms make it difficult to predict the effects of perturbations. For example, strategies for vaccination and quarantine must account for the human-disease feedback, namely, that people's behavior affects the spread of epidemics and epidemics affect people's behavior [1]. Accounting for feedbacks is especially important for controlling systems poised near a critical point because small changes can cause dramatic consequences. Many engineered and natural systemssuch as forest fires [2], power grids [3], water reservoirs [4], brains [5,6], economies [7,8], and financial markets [9]-appear to self-organize toward critical points with power-law-distributed event sizes, a phenomenon called self-organized criticality (SOC). Thus, controlling these systems can profoundly affect systemic risk (i.e., the chance of system-wide catastrophe). For example, suppressing small blackouts in power grids may increase the risk of large ones [3]; making grids "smart" by adding meters, controlling loads, and introducing differential pricing $[10,11]$ could affect reliability in diverse ways. To meet the challenge of controlling self-organizing systems, here we use analytical models that capture the system's feedback mechanisms, i.e., models that self-organize.

Consider a complex system $\mathcal{S}$ that self-organizes to a stationary state $\hat{\mathcal{S}}$. To simplify and understand $\mathcal{S}$, we model $\mathcal{S}$ in one of two ways. An empirical model $\mathcal{E}$ contains parameters measured from the stationary state $\hat{\mathcal{S}}$. By contrast, a mechanistic model $\mathcal{M}$ contains only mechanistic rules (without empirical measurements of $\hat{\mathcal{S}}$ ). Although $\mathcal{E}$ provides insight into $\hat{\mathcal{S}}, \mathcal{E}$ cannot predict the effects of controlling the system away from $\hat{\mathcal{S}}$. However, if $\mathcal{M}$ self-organizes to a stationary state $\widehat{\mathcal{M}}$ via mechanisms like those that drive $\mathcal{S}$ to $\hat{\mathcal{S}}$, then controlling $\mathcal{M}$ to change $\widehat{\mathcal{M}}$ can efficiently predict ways to control $\mathcal{S}$ to change $\hat{\mathcal{S}}$, thus solving the open problems of reducing the systemic risk of and enhancing the function of self-organizing systems $[1-6,9]$.
PACS numbers: $89.75 . \mathrm{Hc}, 02.30 . Y y, 05.65 .+b, 45.70 . \mathrm{Ht}$

In this Letter, we contribute a successful example of controlling an SOC system using a mechanistic model. Here, the system is the BTW sandpile process on a network, denoted by $\mathcal{S}_{\mathrm{BTW}}$, and $\mathcal{E}_{\mathrm{BTW}}$ is a well-studied past model [12]. Our mechanistic model $\mathcal{M}_{\text {BTw }}$ is a multitype branching process that self-organizes by fixing its free parameters via self-consistency. Using $\mathcal{M}_{\mathrm{BTW}}$, we systematically evaluate a control scheme for $\mathcal{S}_{\mathrm{BTW}}$, an exercise expensive to simulate with $\mathcal{S}_{\text {BTW }}$ and impossible with $\mathcal{E}_{\text {BTW }}$. Specifically, we control how often cascades occur, which affects how large cascades could plausibly be. The results illuminate the tradeoffs that plague strategies to control many natural, financial, and infrastructure systems: frequently triggering cascades mitigates large events but sacrifices short-term profit, while avoiding cascades maximizes short-term profit but suffers from rare, massive events. We expect self-organizing, multitype branching processes like $\mathcal{M}_{\mathrm{BTW}}$ to inform controlling other selforganizing systems, including multistate dynamics with information bouncing back-and-forth on networks.

$B T W$ sandpile on a network.-The presence of power laws in the magnitudes of events occurring in many realworld systems is often attributed to SOC [2-6,9,13,14]. Typically, two competing mechanisms dominate: large events slowly but steadily become more probable, whereas the probability of future large events decreases when a large event occurs. For example, tectonic energy builds and then releases in earthquakes [15]. As another example, investment managers or infrastructure stakeholders balance cost and fear: they may slowly increase risk for profit, but when catastrophe occurs they mitigate risk via selfmoderation or imposed regulations.

The BTW sandpile process $[13,14]$ is an archetypal example of such mechanisms. We slowly add grains of sand (interpreted as load) to the system, which increases the chance of large cascades, but grains dissipate (disappear) during cascades. Originally introduced on the 2D lattice $[13,14]$, the BTW sandpile process has since been generalized to networks in a few natural ways that differ 
only in specifics $[12,15-23]$. In this Letter, we consider the following formulation $[12,22,23]$.

The system $\mathcal{S}_{\text {BTw }}$ consists of a network of $N$ nodes that hold grains of sand. The structure of the network is fixed, but the amount of sand on each node changes in time. We call a node $i$-sand if it holds $i$ grains of sand. The capacity of a node is the maximal amount of sand that it can hold. In this Letter, we set the capacity of every node to 1 less than its degree (number of neighbors) [12,19-23]. Hence, a 2 -sand node of degree 3 is at capacity, meaning that it holds as much sand as it can withstand. Adding a grain to this node brings it over capacity. A node over capacity topples, meaning that it sheds its load by sending one grain to each of its neighbors.

The process consists of cascades (avalanches) defined as follows. Drop a grain of sand on a node chosen uniformly at random, called the root of the cascade. If this addition does not bring the root over capacity, then that cascade is finished. Otherwise, the root topples by shedding one grain to each of its neighbors. Any node that now exceeds its capacity topples in the same way, until all nodes are under or at capacity. Whenever a grain of sand moves from one node to another, it dissipates (disappears) independently with probability $\epsilon$. The size of a cascade is the number of toppling events, while the area of a cascade is the number of nodes that topple. We begin a new cascade by dropping a grain on a uniformly random root node. See the Supplemental Material (SM) [24] for the algorithm used in simulations.

In the dual limit of infinite network size $(N \rightarrow \infty)$ and then rare dissipation $(\epsilon \rightarrow 0)$ [25], it has been shown that the system self-organizes to a critical state $\hat{\mathcal{S}}_{\text {BTW }}[12,19]$. Both the cascade area and the cascade size distribution then exhibit a power law with exponent $-\tau$, where $\tau=3 / 2$ for random graphs with light-tailed degree distributions (the mean-field case) and $\tau=\gamma /(\gamma-1)$ for random graphs with power-law degree distributions of exponent $\gamma$ [12].

For simplicity, this Letter considers the BTW process on a random 3-regular graph (i.e., a random network of degree-three nodes). We define $\psi_{0}, \psi_{1}$, and $\psi_{2}$ to be the probabilities that a uniformly random node is $0-, 1-$, or 2 -sand, respectively. Similarly, for all $i, j \in\{0,1,2\}$, we define $\phi_{i j}$ to be the probability of reaching a $j$-sand node by following a link from a uniformly random $i$-sand node. Note that the methods used in this Letter can generalize to networks other than 3-regular, which will be considered in an upcoming publication.

Empirical and self-organizing models.-A simple, empirical model $\mathcal{E}_{\mathrm{BTW}}$ of the BTW sandpile process on a random 3-regular graph may be derived as follows. Assuming $N \rightarrow \infty$, the probability generating function (PGF) for cascade area, $G(x) \equiv \sum_{a=0}^{\infty} \mathbb{P}($ area $=a) x^{a}$, can be obtained by a standard single-type branching process

$$
\begin{aligned}
& F(x)=1-(1-\epsilon) \phi_{22}+(1-\epsilon) \phi_{22} x[F(x)]^{2}, \\
& G(x)=1-\psi_{2}+\psi_{2} x[F(x)]^{3} .
\end{aligned}
$$

Equation (1) uses the empirical observation that in treelike graphs only nodes initially at capacity (i.e., nodes at capacity just before the cascade begins) can topple during this cascade; we rigorously prove this observation using a mechanistic perspective in the Supplemental Material [24]. The PGF $F(x)$ gives the contribution to the area of a node that sends a grain to a neighbor $v$ that has not yet toppled: the grain reaches $v$ with probability $1-\epsilon$, and $v$ is at capacity with probability $\phi_{22}$. If both these events occur, then $v$ topples (factor $x$ ) and sends grains toward its three neighbors, two of which have not yet toppled (factor $\left.[F(x)]^{2}\right)$. In $G(x)$, the root is initially at capacity with probability $\psi_{2}$, in which case it topples (factor $x$ ) and sends a grain toward its three neighbors (factor $[F(x)]^{3}$ ).

For fixed dissipation $\epsilon>0$, we can measure $\psi_{2}$ and $\phi_{22}$ in simulations (i.e., in $\hat{\mathcal{S}}_{\mathrm{BTW}}$ ) and use $\mathcal{E}_{\text {BTW }}$ [Eq. (1)] to approximate the probability distribution of cascade area. However, this empirical model cannot predict the parameters $\psi_{2}$ and $\phi_{22}$ on its own. This lack of closure becomes more problematic if we control the system away from its "natural" observed state $\hat{\mathcal{S}}_{\text {BTW. }}$. A simple mechanistic argument partially solves this problem: with $\langle s\rangle$ denoting the average cascade size, the balance of sand input and average dissipation requires $3 \epsilon\langle s\rangle=1$ (one grain added per cascade; three grains shed by toppling; shed grains dissipate with probability $\epsilon$ ). Assuming finite-size cascades for $\epsilon>0$ [i.e., $(1-\epsilon) \phi_{22}<1 / 2$ ], the expected cascade area satisfies

$$
\langle a\rangle \equiv \sum_{a=0}^{\infty} a \mathbb{P}(\text { area }=a)=G^{\prime}(1)=\frac{\psi_{2}\left[1+(1-\epsilon) \phi_{22}\right]}{1-2(1-\epsilon) \phi_{22}},
$$

and we know from empirical observations that $\langle s\rangle \approx\langle a\rangle$. Hence, the criterion for balancing sand, $3 \epsilon\langle s\rangle=1$, converts the empirical model $\mathcal{E}_{\mathrm{BTW}}$ into an intermediate model $I_{\mathrm{BTW}}$, where mechanistic arguments fix $\phi_{22}$ in terms of $\psi_{2}$. Next, we use $I_{\text {BTW }}$ to explore controlling the system $\mathcal{S}_{\mathrm{BTW}}$. Later, we derive a fully mechanistic, self-organizing model $\mathcal{M}_{\text {BTw }}$ [Eq. (3)] that fixes all unknown parameters.

Control and cost.-Rather than suppressing sandpile cascades in just a specific region of a lattice [26,27] or steering the system to a particular state [28,29], here we control the stationary state $\hat{\mathcal{S}}_{\text {BTW }}$ of $\mathcal{S}_{\text {BTW }}$ to change the risk of small and large cascades. Our controller faces severe constraints: she can neither alter the value of $\epsilon>0$, nor the network, nor the cascade mechanism (unlike in Refs. [30,31]). Instead, the controller can only adjust where the first grain of sand of a cascade tends to land. Using some unspecified method, the controller sets the probability $\mu$ that the first grain lands on a 2 -sand node and hence causes a cascade (of size $\geq 1$ ). This rule defines the system $\mathcal{S}_{\mathrm{BTW}}^{\mu}$, which reduces to the uncontrolled system, $\mathcal{S}_{\mathrm{BTW}}$, when $\mu:=\psi_{2}$. 


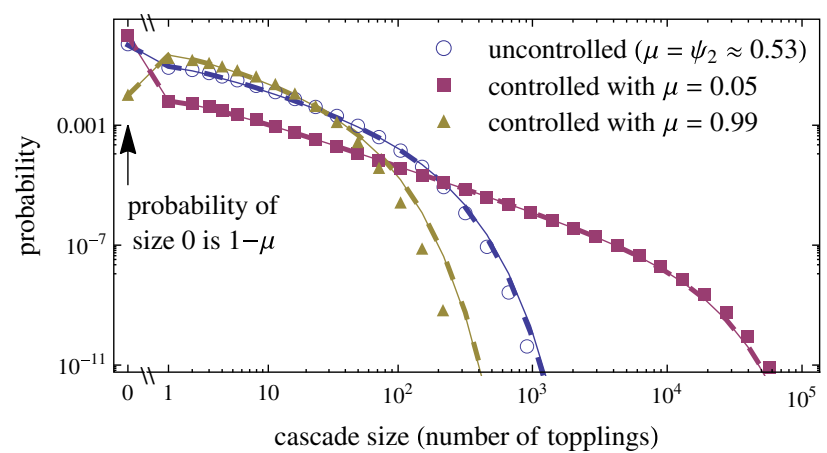

FIG. 1 (color online). Controlling the frequency of cascades $\mu$ significantly affects the cascade size distribution. The chance of no cascade (i.e., a size-zero cascade) is $1-\mu$, while the chance of a cascade of size $\geq 1$ is the control parameter $\mu$. In the original BTW model, $\mu$ is set to $\psi_{2}$, the fraction of 2 -sand (at capacity) nodes. Symbols denote results of simulations on random 3-regular graphs with $\epsilon=0.05, N=10^{6}$, while dashed and plain lines show the predictions of the intermediate model $I_{\mathrm{BTW}}^{\mu}$ and of the self-organizing model $\mathcal{M}_{\mathrm{BTW}}^{\mu}$, respectively.

We obtain the controlled intermediate model $I_{\mathrm{BTW}}^{\mu}$ by substituting $\mu$ for $\psi_{2}$ in $I_{\text {BTw }}$ [i.e., Eqs. (1) and (2) and related], so $\phi_{22}$ is determined by $\epsilon$ and $\mu$. $I_{\mathrm{BTW}}^{\mu}$ can accurately predict the cascade size distribution as one varies $\mu$ (Fig. 1) without needing to observe $\phi_{22}$ empirically, but $I_{\mathrm{BTW}}^{\mu}$ cannot provide $\psi_{2}$, the fraction of 2-sand nodes.

Increasing $\mu$ above $\psi_{2}$ is analogous to dropping snow where avalanches are about to occur and to starting forest fires in fire-prone areas, with the hope of preventing large avalanches and large fires in the long run. This strategy of triggering cascades $\mu=99 \%$ of the time (filled triangles of Fig. 1) does mitigate large avalanches, at the cost of causing more small ones (compared to the uncontrolled case, open circles). The other control strategy, decreasing $\mu$ below $\psi_{2}$, is akin to avoiding cascades as much as possible. Examples include extinguishing all forest fires or engineering power grids to suppress all blackouts, including small ones [3]. This strategy (filled squares of Fig. 1) reduces the frequency of cascades to $\mu=5 \%$, at the cost of making the tail heavier.

The phase diagram of $I_{\mathrm{BTW}}^{\mu}$ (Fig. 2) illustrates the essential behavior: controlling a subcritical system can make it critical. For fixed dissipation $\epsilon$ and control parameter $\mu$, the probability $\phi_{22}$ reaches a steady state (dashed lines). As $\epsilon \rightarrow 0$, the steady state collapses to $\phi_{22}=1 / 2$ for all fixed $\mu \in(0,1]$ and for the uncontrolled system $\left(\mu:=\psi_{2}\right)$. However, for $\epsilon>0$, decreasing $\mu$ brings the system closer to criticality (darker shade of background in Fig. 2) and reaches criticality when $\mu \rightarrow 0$. Thus, decreasing $\mu$ to avoid cascades leads to criticality and hence power-law-distributed event sizes (Fig. 1, squares). By contrast, increasing $\mu$ pushes the stationary state away from the critical line, hence

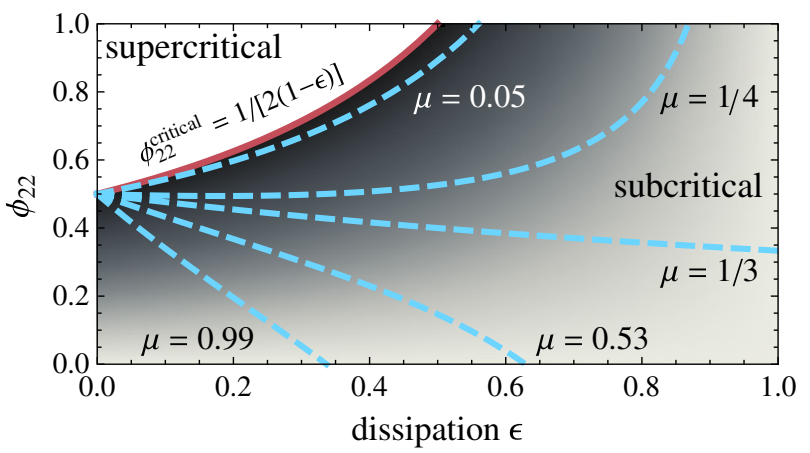

FIG. 2 (color online). Phase diagram of the controlled system $I_{\mathrm{BTW}}^{\mu}$, an approximation of $\mathcal{S}_{\mathrm{BTW}}^{\mu}$ (similar to the diagram in Ref. [34] except with control). Dashed lines are the system's attractors $\phi_{22}=(1-3 \epsilon \mu) /[(1-\epsilon)(3 \epsilon \mu+2)]$. For $\mu>0$, the system is critical only when $\epsilon \rightarrow 0$, but as $\mu \rightarrow 0$ the system approaches the critical line $\phi_{22}^{\text {critical }}=1 /[2(1-\epsilon)]$ for all $\epsilon<1 / 2$. In the subcritical regime, darker shades denote proximity to criticality.

mitigating large cascades (curve $\mu=0.99$ in Fig. 2 and triangles in Fig. 1).

Both $\mu>\psi_{2}$ and $\mu<\psi_{2}$ have tradeoffs, so under what conditions is one strategy better? Because sand input equals average dissipation $(1=3 \epsilon\langle s\rangle)$, we cannot control average cascade size $\langle s\rangle$ using the control parameter $\mu$. However, the cost of a cascade may grow nonlinearly with cascade size, in which case the average cost depends on $\mu$.

Here, we consider two concave cost functions illustrated in Fig. 3 (inset). First, motivated by the idea that small catastrophes in infrastructure are inexpensive to handle but that large disasters become expensive, we define a cost function with a slope $m_{\mathrm{OK}}$ for events smaller than a tipping point $s_{\text {tip }}$ and a steeper slope $m_{\text {bad }}$ for events larger than $s_{\text {tip }}$. Our other cost function grows smoothly as the cascade size

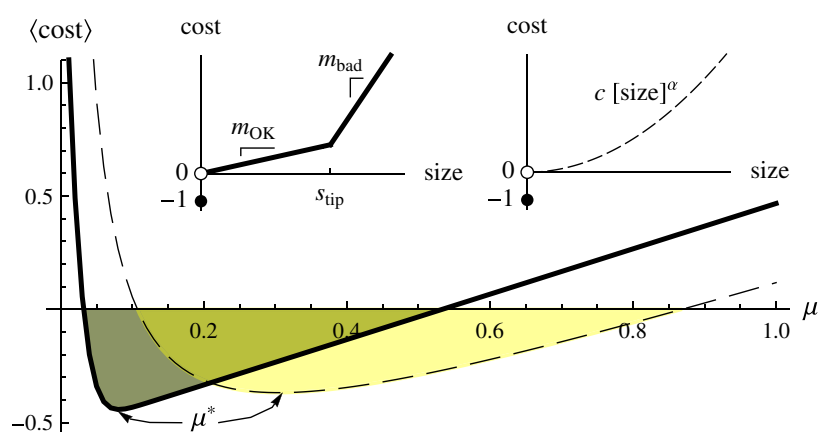

FIG. 3 (color online). If size-zero cascades confer benefit 1 and if, as justified in the text, costs increase nonlinearly, such as with tipping points (left inset, thick lines) or as cascade size raised to a power $\alpha>1$ (right inset, dashed lines), then there may exist a nontrivial, optimal control parameter $\mu^{*}$ that minimizes the expected cost in the stationary state $\hat{\mathcal{S}}_{\mathrm{BTW}}^{\mu}$ of the controlled SOC system $\mathcal{S}_{\mathrm{BTW}}^{\mu}$. (Here, $\boldsymbol{\epsilon}=0.05$ and $m_{\mathrm{OK}}=0.07$, $m_{\text {bad }}=0.5, s_{\text {tip }}=10^{4} ; c=0.005, \alpha=1.5$.) 
raised to a power $\alpha>1$. [Both cost functions could arise from risk aversion (extra disutility to bad outcomes) [32], government penalties for starting cascading failures, herdlike loss of consumer confidence, and/or indirect costs of disasters due to interdependencies with human health and with other infrastructures.] Finally, both cost functions assign a benefit of 1 for size-zero cascades (in which no nodes topple); this benefit defines the scale of costs. (Infrastructures and investment portfolios, for instance, typically profit on uneventful days, yet catastrophes incur costs.)

For many parameters, these two concave cost functions have a nontrivial, optimal control parameter $\mu^{*}$ that minimizes the expected cost of cascades in the stationary state $\hat{\mathcal{S}}_{\text {BTW }}^{\mu}$ (Fig. 3). Increasing $\mu$ above $\mu^{*}$ mitigates large cascades but exacerbates small ones that accrue costs, while decreasing $\mu$ below $\mu^{*}$ makes cascades more rare but enables especially costly, massive cascades. The Supplemental Material shows evidence that optimal $\mu^{*}$ is generic for heavy-tailed event distributions [24].
Finding or avoiding 2-sand nodes becomes difficult when they become rare or widespread, respectively. To model this phenomenon, the controller could use her budget to apply a force $f$ to achieve a $\mu$ given by, e.g., $\mu\left(\psi_{2}, f\right):=\tan ^{-1}\left(f-\cot \left(\pi \psi_{2}\right)\right) / \pi+1 / 2$, so that $f=0$ recovers $\mathcal{S}_{\mathrm{BTW}}$, and pushing $\mu$ to 1 or 0 requires infinite force $f$. However, $I_{\mathrm{BTW}}^{\mu}$ does not provide $\psi_{2}$, so a closed model is needed.

Self-organizing model.-We now introduce a mechanistic, multitype branching process $\mathcal{M}_{\mathrm{BTW}}^{\mu}$ that self-organizes to a stationary state $\widehat{\mathcal{M}}_{\mathrm{BTW}}^{\mu}$ without using empirical measurements of $\mathcal{S}_{\mathrm{BTW}}$. PGFs predict cascade outcome: $w$ and $x$ generate the cascade size and area, respectively, while $y_{i}$ and $z_{i}$ (elements of the vectors $\mathbf{y}$ and $\mathbf{z}$ ) generate the changes in the numbers of $i$-sand nodes and of $i i$-sand links (edges between two $i$-sand nodes), respectively. Unlike $I_{\mathrm{BTW}}^{\mu}$, self-consistency here fixes all parameters $\psi_{i}$ and $\phi_{i j}$ : on average, the numbers of $i$-sand nodes and of $i i$-sand links do not change at the stationary state.

Like $G(x)$ [Eq. (1b)], the PGF $H(w, x, \mathbf{y}, \mathbf{z})$ tracks the contribution of the cascade's root

$$
H=\frac{1-\mu}{1-\psi_{2}} \sum_{i=0}^{1} \psi_{i} \frac{y_{i+1}}{y_{i}}\left[\sum_{j=0}^{2} \phi_{i j} \frac{1+\delta_{(i+1) j}\left(z_{j}-1\right)}{1+\delta_{i j}\left(z_{j}-1\right)}\right]^{3}+\mu x \sum_{n=1}^{\infty} w^{n} \sum_{i^{\prime}=0}^{2} \frac{y_{i^{\prime}}}{y_{i}}\left(\begin{array}{l}
3 \\
i^{\prime}
\end{array}\right)\left[{ }_{n^{\prime}}^{i^{\prime}} A(w, x, \mathbf{y}, \mathbf{z})\right]^{3-i^{\prime}}\left[\begin{array}{l}
i^{\prime} B \\
n_{\hookrightarrow}
\end{array}(w, x, \mathbf{y}, \mathbf{z})\right]^{i^{\prime}}
$$

If the root is initially $i$-sand with $i \in\{0,1\}$ [first term of Eq. (3)], then it becomes $(i+1)$-sand and does not topple. Thus, the network has one fewer $i$-sand node (factor $y_{i}^{-1}$ ) and one more $(i+1)$-sand node (factor $\left.y_{i+1}\right)$. Furthermore, each link between the root and a $j$-sand neighbor warrants a factor $z_{j}^{-1}$ (respectively, $z_{j}$ ) if $j=i$ (respectively, $j=i+1$ ) to account for the lost (respectively, new) $j j$-sand link. Note that only dyadic correlations (i.e., $\phi_{i j}$ ) are considered.

If the root is initially 2-sand [second term of Eq. (3)], then it topples $n \geq 1$ times (factor $x w^{n}$ ) and ends up $i^{\prime}$-sand after the cascade (factor $y_{i^{\prime}} / y_{i}$ ), where $n$ and $i^{\prime}$ depend on the number of grains that the root receives from its neighbors. Hence, a multitype branching process is required to count back-and-forth exchanges. In general, for a parent node $u$ with child $v$, we define a "type" for each combination $\left(n, n^{\prime}, i^{\prime}\right)$ such that, in a particular cascade, $u$ sends (respectively, receives) a total of $n \geq 1$ grains toward (respectively, $n^{\prime}$ grains from) $v$, and, after the cascade, $u$ has $i^{\prime} \in\{0,1,2\}$ grains. The recurrence equation Eq. (1a) becomes a system of equations for two families of functions ${ }_{n}^{i^{\prime}} A$ and ${ }_{n}^{i} B$, corresponding to the cases $n^{\prime}=n-1$ and $n^{\prime}=n$, respectively, which are the only possibilities for treelike graphs (see the proof and full expressions in the Supplemental Material [24]).

Differentiating Eq. (3) with respect to $y_{i}$ and $z_{i}$ and setting all generators to 1 gives $h_{(i)}$ and $\eta_{(i)}$, the average changes in the numbers of $i$-sand nodes and of $i i$-sand links. By hypothesis, the system has reached the stationary state, which provides the constraints $h_{(i)}=\eta_{(i)}=0 \forall i \in$ $\{0,1,2\}$. Because the $\psi_{i}$ are probabilities and the $\phi_{i j}$ are conditional probabilities, they obey the additional constraints $\sum_{i=0}^{2} \psi_{i}=1, \sum_{j=0}^{2} \phi_{i j}=1 \forall i$, and $\psi_{i} \phi_{i j}=$ $\psi_{j} \phi_{j i} \forall i, j$. Starting from educated guesses, numerical solution of the system of constraints provides values of $\psi_{i}$ and $\phi_{i j}$ consistent with those observed in the Monte Carlo simulations of $\mathcal{S}_{\mathrm{BTW}}^{\mu}$, and it enables exploring ranges of parameters that would be computationally costly to simulate (large $N$, low $\epsilon$, and/or low $\mu$; see the Supplemental Material [24]). Values of $\psi_{2}$ obtained in this way may estimate the force $f$ required to achieve some control parameter $\mu\left(\psi_{2}, f\right)$. Finally, the PGF Eq. (3) distinguishes cascade size and area (Fig. 4), which to the best of our knowledge is a new result for BTW cascades on networks.

Future work.-Self-organizing branching processes could enable control of cascade area: if damage must occur, perhaps we can isolate it. For $\mathcal{S}_{\mathrm{BTw}}$ with $\epsilon>0$, a noninvasive control scheme cannot reduce the average cascade size because average sand input must be zero, but our mechanistic understanding of cascades could allow for $\langle$ area/size $\rangle \approx 1 / 2$ in a treelike network and much smaller values in networks containing communities [24].

Adjusting the time scales so that more control occurs between cascades would make this model a dynamic version of highly optimized tolerance (HOT) [33] but with repeated cascades and control. Tuning the time scale 


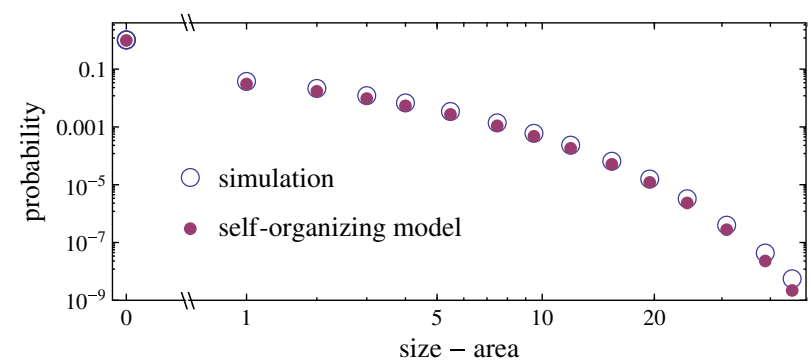

FIG. 4 (color online). Without any parameters from simulations, a self-organizing model can predict quantities inaccessible with past models, such as the area and the size of cascades. Here, we plot the probability distribution for the difference between size and area for a random 3-regular graph with $N=$ $10^{6}, \epsilon=0.001$.

between control and cascades could capture systems ranging from finance and brains (frequent cascades) to power grids and forest fires (infrequent cascades).

The authors thank Kwang-Il Goh for useful discussion. This work was supported in part by the Defense Threat Reduction Agency Basic Research Grant No. HDTRA110-1-0088; the Army Research Laboratory Cooperative Agreement W911NF-09-2-0053; the Department of Defense (DoD) (C. D. B.); and the Fonds de recherche du Québec-Nature et technologies (FRQNT) (P.-A.N.).

*noel.pierre.andre@gmail.com

${ }^{\dagger}$ Also at The Santa Fe Institute, Santa Fe, New Mexico 87501, USA.

[1] N. Perra, D. Balcan, B. Gonçalves, and A. Vespignani, PLoS One 6, e23084 (2011).

[2] B. D. Malamud, G. Morein, and D. L. Turcotte, Science 281, 1840 (1998).

[3] I. Dobson, B. A. Carreras, V. E. Lynch, and D. E. Newman, Chaos 17, 026103 (2007).

[4] G. L. Mamede, N. A. M. Araujo, C. M. Schneider, J. C. de Araújo, and H. J. Herrmann, Proc. Natl. Acad. Sci. U.S.A. 109, 7191 (2012).

[5] T. Ribeiro, M. Copelli, F. Caixeta, and H. Belchior, PLoS One 5, e14129 (2010).

[6] A. Haimovici, E. Tagliazucchi, P. Balenzuela, and D. R. Chialvo, Phys. Rev. Lett. 110, 178101 (2013).

[7] P. Bak, K. Chen, J. Scheinkman, and M. Woodford, Ricerche Economiche 47, 3 (1993).

[8] S.F. Nørrelykke and P. Bak, Phys. Rev. E 65, 036147 (2002).

[9] B. Dupoyet, H. Fiebig, and D. Musgrove, Physica (Amsterdam) 390A, 3120 (2011).

[10] K. Moslehi and R. Kumar, IEEE T. Smart Grid 1, 57 (2010).

[11] S. Blumsack and A. Fernandez, Energy 37, 61 (2012).
[12] K.-I. Goh, D.-S. Lee, B. Kahng, and D. Kim, Phys. Rev. Lett. 91, 148701 (2003).

[13] P. Bak, C. Tang, and K. Wiesenfeld, Phys. Rev. Lett. 59, 381 (1987).

[14] P. Bak, C. Tang, and K. Wiesenfeld, Phys. Rev. A 38, 364 (1988).

[15] S. Lise and M. Paczuski, Phys. Rev. Lett. 88, 228301 (2002).

[16] L. de Arcangelis and H. J. Herrmann, Physica (Amsterdam) 308A, 545 (2002).

[17] M. Hoore and S. Moghimi-Araghi, J. Phys. A 46, 195001 (2013).

[18] J. Lahtinen, J. Kertész, and K. Kaski, Physica (Amsterdam) 349A, 535 (2005).

[19] E. Bonabeau, J. Phys. Soc. Jpn. 64, 327 (1995).

[20] D.-S. Lee, K.-I. Goh, B. Kahng, and D. Kim, Physica (Amsterdam) 338A, 84 (2004).

[21] K.-I. Goh, D.-S. Lee, B. Kahng, and D. Kim, Physica (Amsterdam) 346A, 93 (2005).

[22] K.-M. Lee, K.-I. Goh, and I. M. Kim, J. Korean Phys. Soc. 60, 641 (2012).

[23] C. D. Brummitt, R. M. D'Souza, and E. A. Leicht, Proc. Natl. Acad. Sci. U.S.A. 109, E680 (2012).

[24] See Supplemental Material at http://link.aps.org/ supplemental/10.1103/PhysRevLett.111.078701 for details concerning the simulation algorithm, the cost analysis in a generic controlled SOC context, the mechanistic approach, and opportunities for controlling the cascade area.

[25] Contrast (i) this dual limit $N \rightarrow \infty, \epsilon \rightarrow 0$ for the BTW process on a network with (ii) the single limit $N \rightarrow \infty$ for traditional formulations of the BTW process on a twodimensional lattice. In (ii), sand dissipates upon being shed off the boundary of the lattice, and the relative size of the boundary to the whole lattice vanishes in the limit $N \rightarrow \infty$. Hence, the single limit in (ii) implicitly achieves vanishing dissipation comparable to the limit $\epsilon \rightarrow 0$ in (i).

[26] D. O. Cajueiro and R.F. S. Andrade, Phys. Rev. E 81, 015102(R) (2010).

[27] D. O. Cajueiro and R.F. S. Andrade, Phys. Rev. E 82, 031108 (2010).

[28] Y.-Y. Liu, J.-J. Slotine, and A. L. Barabási, Nature (London) 473, 167 (2011).

[29] S.P. Cornelius, W.L. Kath, and A.E. Motter, Nat. Commun. 4, 1942 (2013).

[30] D. O. Cajueiro and R. F. S. Andrade, Eur. Phys. J. B 77, 291 (2010).

[31] G. D’Agostino, A. Scala, V. Zlatić, and G. Caldarelli, Europhys. Lett. 97, 68006 (2012).

[32] M. E. J. Newman, M. Girvan, and J. D. Farmer, Phys. Rev. Lett. 89, 028301 (2002).

[33] J. M. Carlson and J. Doyle, Phys. Rev. Lett. 84, 2529 (2000).

[34] K. B. Lauritsen, S. Zapperi, and H. E. Stanley, Phys. Rev. E 54, 2483 (1996). 